\title{
Fever and a rat bite
}

\author{
Christopher M Booth MD, Kevin C Katz MD FRCPC, James Brunton MD FRCPC
}

A 70-year-old previously healthy man presented to the emergency department with a three-day history of fever. Two weeks before, while working in the kitchen of his restaurant, he captured a rat. The rat bit him on his right thumb, prompting the man to kill the rat and dispose of it in the garbage. He saw his family physician, who gave him a topical antibiotic ointment and a tetanus booster. The wound healed and the patient felt well until 10 days later when he developed fever, chills and sweats. At the same time, his thumb became painful, swollen and erythematous. The wound dehisced spontaneously. He returned to his family physician, who prescribed oral cloxacillin and referred him to the emergency department.

Upon arrival at the emergency department, his temperature was $39.1{ }^{\circ} \mathrm{C}$ and his oxygen saturation was $90 \%$ on room air. Blood and wound swab cultures were obtained. The patient was given a second tetanus booster and a consultation with the infectious diseases service was requested.

The patient was from China and had lived in Canada since 1996. He had not travelled outside Canada since his arrival. His vaccination history was unknown. There was no history of arthralgias, myalgias or rash.

On physical examination by the infectious disease service, his blood pressure was $110 / 80 \mathrm{mmHg}$, his heart rate was 70 beats/min and regular, and his temperature was $36.7^{\circ} \mathrm{C}$. His oxygen saturation was $97 \%$ on room air. There was no cervical adenopathy and his conjunctiva were normal. His neck was supple. Cardiovascular and abdominal examinations were normal. Auscultation of his chest revealed bibasilar inspiratory crackles. There was no rash, petechiae, jaundice or clubbing. His right thumb was swollen and erythematous to the base. There was a superficial puncture

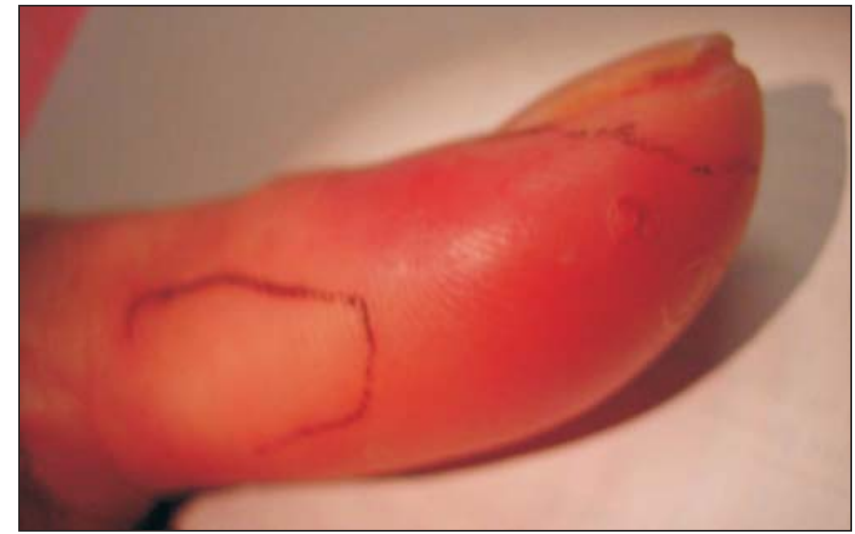

Figure 1) Erythematous thumb with margins demarcated and open puncture site

wound on the ventromedial aspect of his thumb (Figure 1). There was no drainage. His thumb was tender and indurated. There was also evidence of lymphangitis on the dorsal aspect of his right forearm.

Initial laboratory investigations revealed a white blood cell count of $9.5 \times 10^{9} / \mathrm{L}$, and normal electrolyte, renal and liver profiles. Two sets of blood cultures were negative. A Gram stain of the sample from the wound showed Gramnegative bacilli on microscopy, but the culture was negative. Radiographs of his chest and hand were normal.

What is your diagnosis and how would you treat this patient?

continued on page 271 


\section{CLINICAL VIGNETTE}

continued from page 269

\section{DIAGNOSIS}

A diagnosis of rat bite fever was made and the patient was treated with 14 days of amoxicillin/clavulinic acid. He became afebrile and was discharged $48 \mathrm{~h}$ after starting antibiotics, at which time the erythema, pain and edema in his right thumb had improved and the lymphangitis had resolved. At follow-up two weeks later, he felt generally well and his thumb was not swollen or tender, but there was some residual erythema and induration surrounding the area of the bite. The initial blood cultures (taken $24 \mathrm{~h}$ after starting oral cloxacillin) and wound swab culture were negative. Universal bacterial $16 \mathrm{~S}$ ribosomal RNA polymerase chain reaction of the wound swab was negative.

\section{DISCUSSION}

Rat bite fever is a rare but well described clinical syndrome. It is caused by two different pathogens that are commonly found in the oropharynx of rodents - Streptobacillus moniliformis and Spirillum minus. Originally described as 'sodoku' in Japan, cases of rat bite fever have been reported worldwide.

$S$ moniliformis is a microaerophilic, Gram-negative bacillus that accounts for most cases of rat bite fever in North America (1). It was previously known as Haverhilia multiformis, named after the epidemic of rat bite fever that occurred in Haverhill, Massachusetts in 1926 (2). S minus (also a Gram-negative bacillus) accounts for most cases of rat bite fever in Asia. Artificially induced spirillar fever, or 'sodoku inoculata', was used in the 1930s for the treatment of dementia paralytica (neurosyphillis). Affected individuals were inoculated with blood from guinea pigs and developed symptomatology that resembled that of clinical rat bite fever (3).

Rat bite fever has been reported in a number of populations, including urban poor communities, rural communities, laboratory workers and children with pets (1). The infection typically follows a rat bite or scratch, although cases have been reported from contact with squirrels, mice, dogs and cats (4). Furthermore, rat bite fever can follow the ingestion of food or water that has been contaminated with rat excrement. This was demonstrated by the outbreak of rat bite fever in Haverhill, Massachusetts in 1926, when a contaminated milk source led to infection in 86 individuals (2).

The clinical features of this syndrome typically appear one to two weeks after the initial bite. Patients report an initial asymptomatic period followed by the abrupt onset of fever, chills, nausea and headache. Many people also develop myalgias and arthralgias. A maculopapular or petechial rash may form on the soles of the feet or palms of the hands of affected individuals. If untreated, the symptoms resolve
TABLE 1

Clinical features of rat bite fever

\begin{tabular}{ll}
\hline Streptobacillus monoliformis & Spirullum minus \\
\hline North America & Asia \\
Onset within 10 days & Onset in one to four weeks \\
Wound heals, inapparent & Wound inflamed, reopens \\
Arthralgias common & Arthralgias uncommon \\
No lymphangitis & Lymphangitis \\
Rash on hands and feet & Typically no rash \\
\hline
\end{tabular}

spontaneously within days, although relapses may occur weeks to months later. Rare complications of rat bite fever include endocarditis, pneumonia, septic arthritis, myocarditis, amnionitis and abscess formation. The mortality rate of untreated patients has been reported to be $13 \%$ (4). S moniliformis is almost the exclusive cause of rat bite fever in North America and Europe, while $S$ minus is responsible for rat bite fever in Asia. The amount of time from bite to the onset of symptoms tends to be longer in cases of $S$ minus. Other distinguishing features include the presence of rash, arthralgia and myalgia in Streptobacillus infections. Spirillum infections are characterized by reopening and eventual ulceration of the wound after it has healed, as well as regional lymphangitis and lymphadenitis (Table 1) (5). The present case is more typical of $S$ minus than $S$ moniliformis. It is possible, however, that the presenting syndrome was caused by a staphylococcal or streptococcal species that frequently causes skin and soft tissue infections.

Diagnosis of rat bite fever relies largely on history and physical examination. Laboratory diagnosis is frequently difficult. Streptobacillus requires culturing on enriched media, and Spirillum does not grow on culture media. Spirillum can be diagnosed only by direct visualization of the typical motile organisms. Both organisms are susceptible to penicillin, and the recommended treatment includes a 10 to 14-day course of antibiotics along with suitable tetanus prophylaxis. Postexposure rabies prophylaxis is not recommended for the treatment of rat bites (6).

Most cases of rat bite fever reported in the North American literature were caused by $S$ moniliformis, the majority of which occured in laboratory workers, pet owners or people who lived in areas that were infested with rats. It is estimated that $50 \%$ of laboratory and wild rats in North America harbour Streptobacillus in the oropharynx (7). The most recently reported case of rat bite fever in Canada occurred in 1964 in a female graduate student who was bitten by a laboratory rat (8). Streptobacillus was eventually cultured on enriched media. In 1951, two cases of rat bite fever were reported in children who lived together in urban Vancouver, British Columbia and who had been bit- 
ten by a rat. Streptobacillus was cultured from the blood of both patients. The symptoms recurred periodically for several months. There were eight other cases of rat bite fever reported in Canada before 1940. Seven of those cases occurred in urban Montreal, Quebec (mostly in children), and the other case occurred in rural Nova Scotia. Cultures

\section{REFERENCES}

1. Anderson LC, Leary SL, Manning PJ. Rat bite fever in animal research laboratory personnel. Lab Anim Sci 1983;33:292-4.

2. Parker F, Hudson NP. The etiology of Haverhill fever. Am J Pathol 1926;2:357-79.

3. Solomon HC, Berk A, Theiler M, et al. Arch Int Med 1926;38:391.

4. Gunning JJ. Rat bite fevers. In: Hunter GW, Swartzwelder JC, Clyde DF, eds. Tropical Medicine, 5th edn. Philadelphia: WB Saunders, 1976:246-7.

5. Roughgarde JW. Antimicrobial therapy of rat bite fever. Arch Intern Med 1965;39:116. did not reveal any pathogenic organisms, although in three cases, $S$ minus was reportedly visualized in the blood (9).

We describe the first reported case of rat bite fever in Canada since 1964. This distinct clinical syndrome should be considered in anyone who presents with fever and may be at risk of exposure to rats.

6. Mandell GL, Douglas RG Jr, Bennett JE, eds. Principles and Practice of Infectious Diseases, 5th edn. New York:

Churchill Livingstone, 2000.

7. Strangeways WT. Rats as carriers of Streptobacillus moniliformis. J Pathol Bacteriol 1933;116:39.

8. Houlden FA, MacKay JC. Rat bite fever - An occupational hazard. CMAJ 1964;91:78-81.

9. Dolman CE, Kerr DE, Chang H, et al. Two cases of rat bite fever due to Streptobacillus monoliformis. Can J Pub Health 1951;42:228-41. 


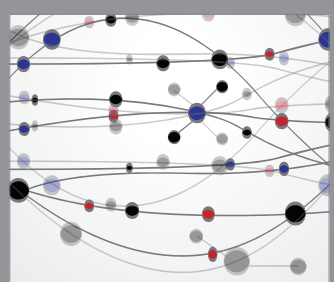

The Scientific World Journal
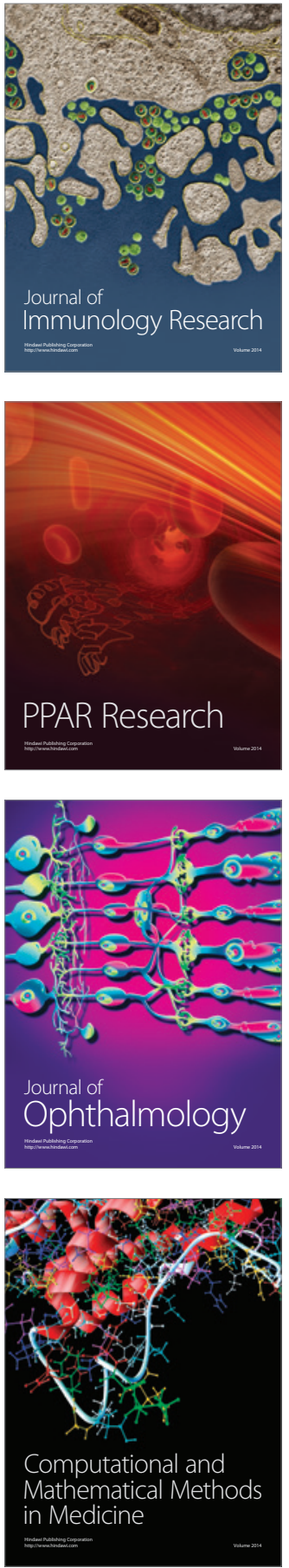

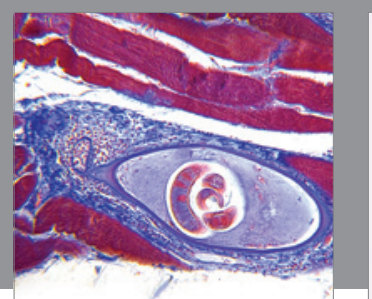

Gastroenterology Research and Practice

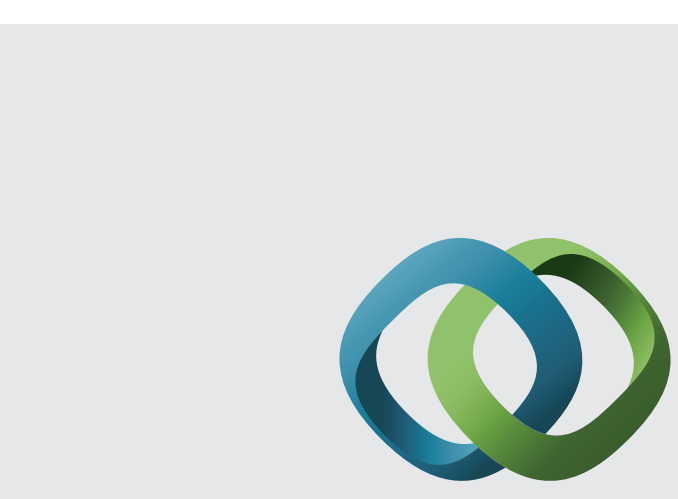

\section{Hindawi}

Submit your manuscripts at

http://www.hindawi.com
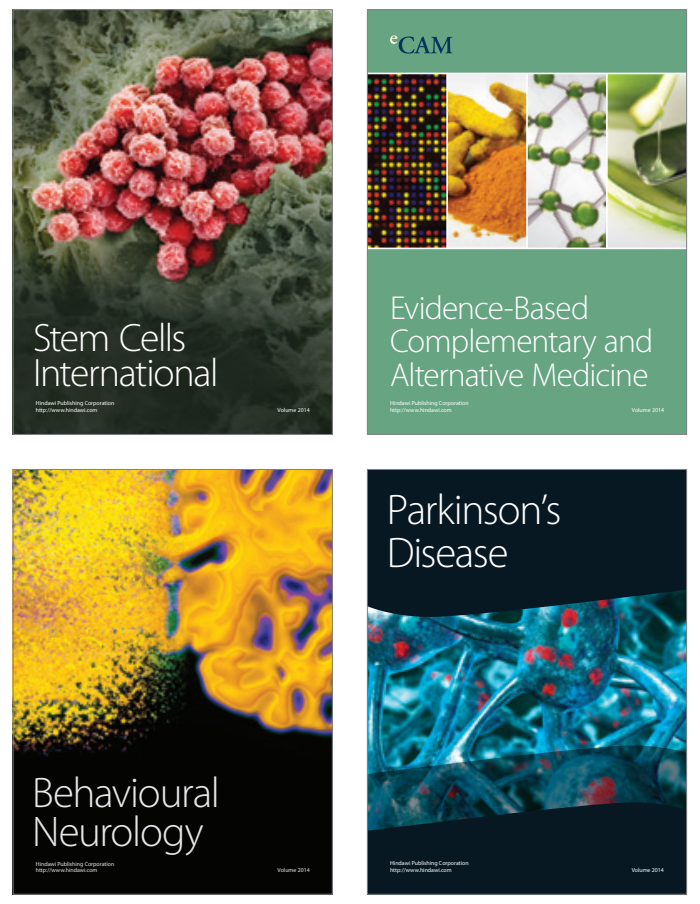
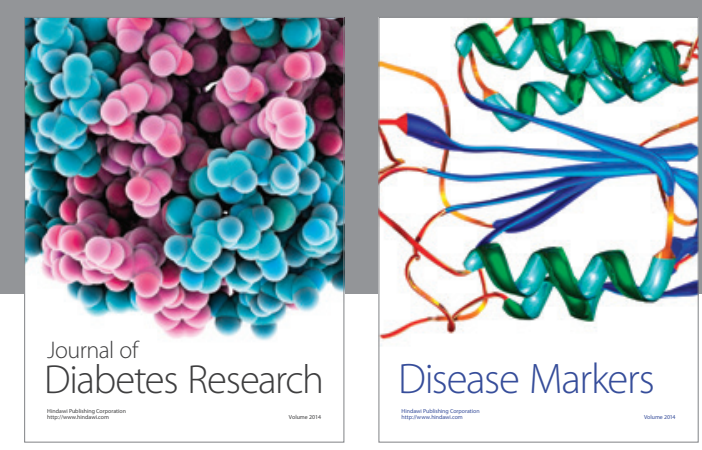

Disease Markers
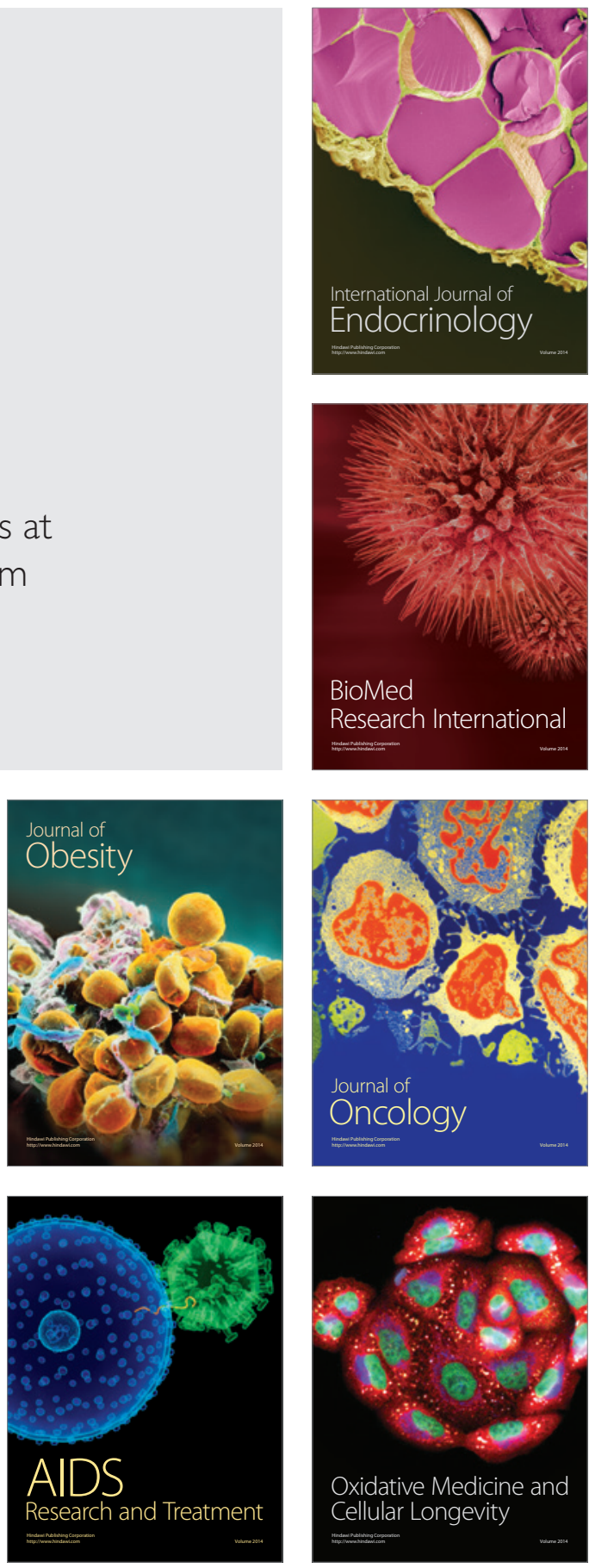